\title{
Azimuthal decorrelations of dijets in QCD
}

\author{
Andrea Banfi ${ }^{1}$ \\ 1- Università di Milano-Bicocca and INFN, Sezione di Milano-Bicocca \\ Piazza della Scienza 3, 20126 Milano - Italy
}

\begin{abstract}
We report on the status of the QCD analysis of dijet azimuthal decorrelations. We emphasise the relevance of resummation of soft and collinear enhancements in describing these observables in the region where the two jets are nearly back-to-back in the transverse plane. We also discuss the sources of theoretical uncertainties and possible research directions aimed at their reduction.
\end{abstract}

\section{Dijet azimuthal decorrelations}

The study of jet cross sections constitutes one of the most important investigation tools for the physics of hadronic final states. These observables are favoured in many aspects, both theoretically and experimentally. First they are infrared and collinear safe a which makes it possible to compute them at all orders in QCD. Hadronisation corrections are quite small, being suppressed by inverse powers of the transverse energy of the jets, and their dependence on the jet radius $R$ is under control [2]. Methods to eliminate the contamination of underlying event and pile-up are also being developed [3]. Furthermore, measurements involving jets show less experimental uncertainties with respect to the corresponding ones involving particles (see for instance Ref. 4, for a recent analysis).

Among jet observables we consider the azimuthal decorrelation of a pair of jets [5, 6 . At tree level two jets produced in a hard collision are highly correlated, their transverse momenta with respect to the beam being exactly back-to-back, which implies that their relative azimuthal angle $\Delta \phi$ is equal to $\pi$. Additional QCD radiation decorrelates the dijet system, moving $\Delta \phi$ away from its Born value. This feature, together with the fact that any measurement involving angles, i.e. ratios of momenta, is less sensitive to jet energy scale uncertainties, make azimuthal decorrelations an ideal ground to explore all-order properties of QCD dynamics. In particular, in the region where $\Delta \phi$ is close to $\pi$, we expect the dominance of configurations with multiple soft and collinear emissions. On the contrary, when $\Delta \phi$ is small, if the available rapidity range is large enough, one may expect the occurrence of multiple hard emissions decorrelating the two jets. Besides these perturbative features, azimuthal decorrelations are also affected by intrinsic transverse momentum of partons inside the incoming hadrons, thus making it possible to explore this non-perturbative aspect of QCD.

As far as the experimental situation is concerned, dijet azimuthal decorrelations have been measured both at the Tevatron [5] and at HERA [6]. In hadronic collisions, one observes that next-to-leading order (NLO) QCD predictions obtained with NLOJET++ 7 fail for $\Delta \phi$ near $\pi$. This region is instead described quite well by the Monte Carlo event generators HERWIG [8] and PYTHIA 9]. This reveals that at the Tevatron, where the momentum fraction $x$ of struck partons is quite large, multiple soft and collinear emissions are able to account for the azimuthal decorrelations in the quasi-back-to-back-region. Furthermore, due

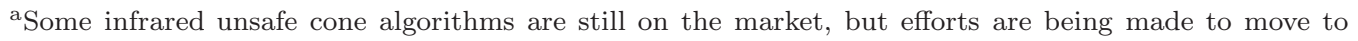
infrared safe jet definitions [1].
}

DIS 2009 
to the sensitivity of Monte Carlo predictions to changes in the shower evolution parameters, these observables should be exploited for the tuning of event generators [5]. HERA instead [6] explored the region of small $x$, where large deviations from Monte Carlo predictions have been observed. However, one finds better agreement with NLO, except of course in the region $\Delta \phi \simeq \pi$. A question then arises on whether the Monte Carlo tuning performed at the Tevatron at large $x$ will be able to describe LHC data which involve smaller values of $x$. An attempt to predict azimuthal decorrelations both at large and small $\Delta \phi$ is represented by CASCADE [10, an event generator producing hard QCD emissions according to the CCFM equation 11. CASCADE, as expected, describes the azimuthal decorrelation in the region of small $\Delta \phi$, while fixing the distribution at $\Delta \phi$ close to $\pi$ by means of an unintegrated parton distribution containing information on the transverse momentum of the incoming parton inside the proton.

We decided to take another point of view. First we observe that a resummation of soft and collinear emissions gives rise to a distribution that rises to a constant value for $\Delta \phi \rightarrow \pi$, consistent with what is seen in the data [5, 6. Moreover, the agreement of NLO QCD with data in the region of small $\Delta \phi$ suggests that in the $x$ region probed at HERA only few extra hard emissions could be considered. It is then reasonable to investigate whether HERA data can be described by a resummation of soft/collinear enhancements matched to exact NLO.

\section{Resummation of soft and collinear logarithms}

The resummation of soft and collinear logarithms is performed by considering the observable $\Sigma(\Delta)$, the probability that $|\pi-\Delta \phi|<\Delta$. The azimuthal decorrelation can be then obtained by differentiating $\Sigma(\Delta)$. The rate $\Sigma(\Delta)$ contains logarithmic contributions up to $\alpha_{s}^{n} \ln ^{2 n} \Delta$, which become large in the back-to-back region $\Delta \ll 1$, and have to be resummed at all orders. The first step to perform such a resummation is to investigate the behaviour of $|\pi-\Delta \phi|$ after a single soft emission $k$, collinear to each of the hard legs (one or two incoming and two outgoing). If $k$ is collinear to any of the incoming partons, we have

$$
|\pi-\Delta \phi| \simeq \frac{k_{t}}{p_{t, 1}}|\sin \phi|
$$

where $k_{t}$ is the emission's transverse momentum (with respect to the beam in hadronic collisions or the virtual photon momentum in DIS), $\phi$ its azimuthal angle, and $p_{t, 1}$ is the transverse momentum of the highest- $p_{t}$ jet. This result does not depend on either the jet algorithm or the recombination scheme of particles within a jet. If instead one considers an emission collinear to one of the outgoing legs, in any jet algorithm, soft gluon $k$ and its parent parton will be clustered in the same jet, and the result will depend on the recombination scheme. In any scheme that adds three-momenta vectorially ( $E$-scheme, $P$-scheme, $E 0$ scheme) the azimuth of the jet does not change, so that $|\pi-\Delta \phi|$ is completely unaffected by emissions inside the hard jets. Therefore the azimuthal decorrelation turns out to be a nonglobal observable [12, its resummation becomes extremely tricky and will not be discussed here. If instead one uses a $p_{t}$-weighted recombination scheme, as is done at HERA, we have

$$
|\pi-\Delta \phi| \simeq \frac{k_{t}}{p_{t, 1}}|\sin \phi-\phi|, \quad i \in \text { jet1; } \quad|\pi-\Delta \phi| \simeq \frac{k_{t}}{p_{t, 1}}|\sin \phi-(\pi-\phi)|, \quad i \in \text { jet2. }
$$


For an arbitrary set of secondary soft and collinear emissions, we have [13]

$$
|\pi-\Delta \phi| \simeq\left|\sum_{i \notin \mathrm{jets}} \frac{k_{t i}}{p_{t, 1}} \sin \phi_{i}-\sum_{i \in \mathrm{jet} 1} \frac{k_{t i}}{p_{t, 1}}\left[\phi_{i}-\sin \phi_{i}\right]-\sum_{i \in \mathrm{jet} 2} \frac{k_{t i}}{p_{t, 1}}\left[\left(\pi-\phi_{i}\right)-\sin \phi_{i}\right]\right|,
$$

and the observable is now global. We stress that the choice of the $p_{t}$-weighted scheme is observable specific. For other observables a different choice could be needed in order to achieve the best theoretical accuracy.

Resummation is better performed in impact parameter space, where the result for $\Sigma(\Delta)$ schematically reads $[13]$

$$
\Sigma(\Delta)=\frac{2}{\pi} \int_{0}^{\infty} \frac{d b}{b} \sin (b \bar{\Delta})\left[\prod_{a=1}^{n_{\text {in }}} \frac{f_{a}\left(\mu_{F} / b\right)}{f_{a}\left(\mu_{F}\right)}\right] e^{-R_{\mathrm{in}}(b)} e^{-R_{\mathrm{out}}(b)} S(b), \quad \bar{\Delta}=\Delta e^{-\gamma_{E}} .
$$

Each function in the above equation accommodates all-order real or virtual corrections in selected phase space regions. The ratios of parton densities $f_{a}\left(\mu_{F} / b\right) / f_{a}\left(\mu_{F}\right)$ embody real and virtual contributions up to the scale $\mu_{F} / b$, above which real emissions are forbidden by the observable definition. All remaining virtual corrections are included in the radiator functions $R_{\text {in }}(b), R_{\text {out }}(b)$, containing virtual corrections collinear to incoming and outgoing legs respectively, and the soft function $S(b)$, accounting for soft large-angle gluons. The above resummation is valid within next-to-leading logarithmic (NLL) accuracy, i.e. accounts for all terms $\alpha_{s}^{n} \ln ^{n+1} b$ and $\alpha_{s}^{n} \ln ^{n} b$ in the logarithm of the Fourier transform of $\Sigma(\Delta)$. At first order in $\alpha_{s}$ the expressions for $R_{\mathrm{in}}(b)$ and $R_{\text {out }}(b)$ read

$$
R_{\text {in }}(b)=C_{\text {in }} \frac{\alpha_{s}}{\pi} \ln ^{2} b, \quad R_{\text {out }}(b)=\frac{C_{\text {out }}}{3} \frac{\alpha_{s}}{\pi} \ln ^{2} b,
$$

where $C_{\text {in }}$ and $C_{\text {out }}$ are the total colour charges of incoming and outgoing legs respectively Note that $R_{\text {in }}(b)$ is the usual radiator for $p_{T}$ resummation (e.g. Drell-Yan lepton-pair $p_{T}$ [14] while the expression for $R_{\text {out }}(b)$ has never been encountered before and reflects the unusual dependence of $|\pi-\Delta \phi|$ on the rapidity and azimuth of each gluon emitted from outgoing legs. The soft function $S(b)$ is in general a matrix in the colour space spanned by the hard emitting partons, and depends on emitters' four-velocities.

\section{Towards phenomenology}

We consider azimuthal dijet decorrelation in DIS $\$$ with the same kinematical cuts adopted by $\mathrm{H} 1$ [6], that is two jets with $-1<\eta^{\text {lab }}<2.5$ and transverse momenta in the hadronic centreof-mass $(\mathrm{HCM})$ frame $p_{t}>5 \mathrm{GeV}$. We give predictions for the differential cross section in the angular distance $\Delta \phi$ in the HCM frame. Results are shown in Fig. 1 for a selected value of $x_{B}$ and $Q^{2}$, reported in the figure. One can see that around $\Delta \phi \simeq 170^{\circ}$ both LO and NLO predictions obtained from NLOJET ++ diverge due to the presence of large logarithms. The NLL resummed curve in Eq. (11) instead rises to a constant value, in qualitative agreement with what is seen in the data [6].

\footnotetext{
${ }^{\mathrm{b}}$ For instance in DIS with an incoming quark $C_{\mathrm{in}}=C_{F}$ and $C_{\text {out }}=C_{F}+C_{A}$.

${ }^{c}$ This observable, defined with a $p_{t}$-weighted recombination scheme, is global. This is not so for the corresponding observable considered at the Tevatron, which is non-global [5].
}

DIS 2009 


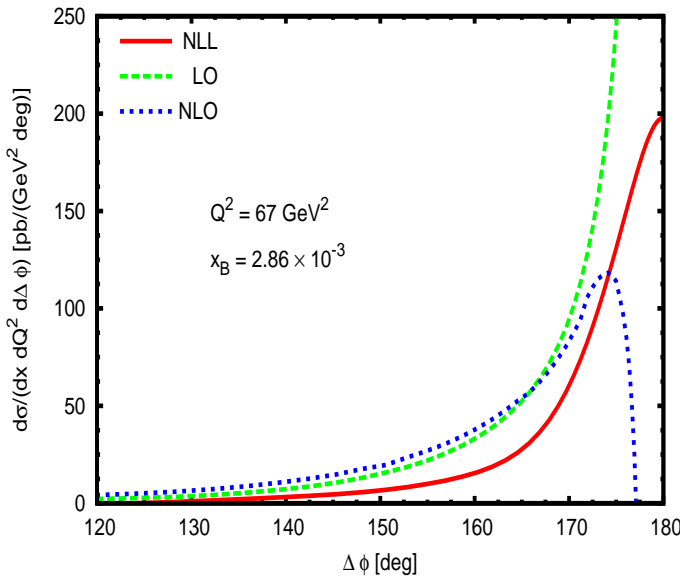

Figure 1: Fixed order and resummed predictions for azimuthal dijet decorrelation in DIS.
The resummed expression in Eq. (1) contains some ambiguities that have to be addressed before comparing to data. First all functions of $b$ in the Fourier transform of $\Sigma(\Delta)$ result from integrals of the running coupling $\alpha_{s}\left(k_{t}\right)$ down to $k_{t}=p_{t, 1} / b$. When $b$ becomes large, this value can exceed the Landau pole, thus making Eq. (1) ill-defined. We then decided to cut the $b$ integral at the value $b_{\max }$ corresponding exactly to the Landau pole. However, since the $b$-integral is strongly suppressed in the large- $b$ region, it is not affected significantly by reducing $b_{\max }$ by a factor, as can be seen in Fig. 2 comparing the curves labelled $b<b_{\max }$ and $b<b_{\max } / 2$. This means that even in the region $\Delta \phi \simeq 180^{\circ}$ dynamics is not dominated by low momentum scales. At small $b$ one has to take into account that setting $b=0$ corresponds to computing the total cross section. This imposes the requirement that the Fourier transform of $\Sigma(\Delta)$ tend to unity as $b \rightarrow 0$. This is achieved by either freezing its value at one for $b<1$ (this is what is done in the plot in Fig. 1) or making the replacement $b \rightarrow \sqrt{1+b^{2}}$ 14. Figure 2 shows that either choice (corresponding to the curves $b<b_{\max }$ and $\left.b \rightarrow\left(1+b^{2}\right)^{1 / 2}\right)$ produces almost indistinguishable results.

The most important source of theoretical errors is the treatment of $f_{a}\left(\mu_{F} / b\right)$, since the actual factorisation scale $\mu_{F} / b$ can be smaller than the inverse size of the proton $R_{p}^{-1}$. The curve in Fig. 1 has been obtained by freezing the parton densities for $b>\mu_{F} R_{p}$. However, restricting the $b$ integral to $b<\mu_{F} R_{p}$ (see Fig. 2) gives a result that can be $50 \%$ smaller in the large $\Delta \phi$ region. This suggests that to provide accurate predictions for azimuthal decorrelations in DIS one may need to consider the effect of the intrinsic transverse momentum of partons inside the proton.

Finally, in order to obtain quantitative predictions, one has to match NLL resummation to exact fixed order calculations. After matching, $\Sigma(\Delta)$ gets multiplied by a coefficient function $\left(1+C_{1} \alpha_{s}+\ldots\right)$ in

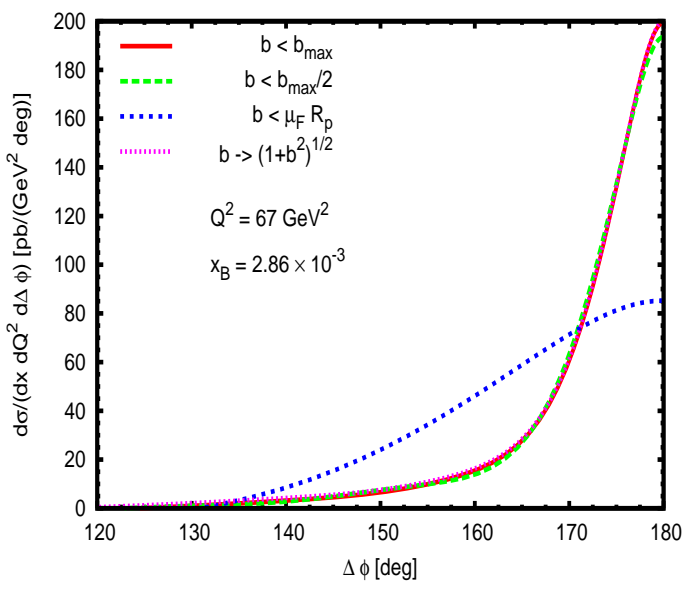

Figure 2: The impact of different prescriptions on azimuthal dijet decorrelations in DIS. such a way that in its expansion all terms $\alpha_{s}^{n} \ln ^{2 n-2} \Delta$ are correctly taken into account. The determination of $C_{1}$ poses two theoretical problems. The first is that, in order to achieve such an accuracy, one has to be able to compute $C_{1}$ for each hard colour configuration. This can be done in the soft/collinear limit 
via a flavour jet-algorithm [15], but requires that one modify NLOJET ++ to include the information on the flavour of produced partons, as has been done in hadron-hadron collisions in Ref. [16. This work is currently in progress. A second issue is the fact that, after subtracting all logarithms from the LO prediction, the result tends to a constant value which is large and negative, thus potentially giving a negative coefficient function. This reveals that probably part of the coefficient function will have to be resummed and eventually exponentiated, as has been done for the Drell-Yan total cross section [17. Potentially large terms may contain $\pi^{2}$, coming either from Coulomb phases or from azimuthal integrations, or large logarithms of Bjorken $x$. The need for better understanding of the coefficient function is one of the reasons why we have decided to study these sub-leading contributions in the simpler case of the $Z$ boson $a_{T}$ distribution in hadron-hadron collisions [18. The resummation for this observable is very similar to that for azimuthal decorrelations, with the simplification that it does not involve any outgoing jets and that it should have no contributions that are logarithmically enhanced by kinematics (e.g. by $\ln 1 / x$ ). These last contributions could be addressed by extending soft collinear resummation to include also multiple hard gluon emissions as is done for instance in CASCADE.

\section{Acknowledgements}

It has been a pleasure to work on this subject together with Mrinal Dasgupta and Yazid Delenda. I am also grateful to the organisers for the cheerful and stimulating atmosphere I have experienced during the whole workshop.

\section{References}

[1] G. P. Salam and G. Soyez, JHEP 0705, 086 (2007) arXiv:0704.0292 [hep-ph]].

[2] M. Dasgupta, L. Magnea and G. P. Salam, JHEP 0802, 055 (2008) arXiv:0712.3014 [hep-ph]].

[3] M. Cacciari, G. P. Salam and G. Soyez, JHEP 0804, 005 (2008) arXiv:0802.1188 [hep-ph]].

[4] G. Dissertori, F. Moortgat and M. A. Weber, arXiv:0810.3208 [hep-ph].

[5] V. M. Abazov et al. [D0 Collaboration], Phys. Rev. Lett. 94, 221801 (2005) arXiv:hep-ex/0409040.

[6] A. Aktas et al. [H1 Collaboration], Eur. Phys. J. C33, 477 (2004) arXiv:hep-ex/0310019]; S. Chekanov et al. [ZEUS Collaboration], Nucl. Phys. B786, 152 (2007) arXiv:0705.1931 [hep-ex]].

[7] Z. Nagy and Z. Trocsanyi, Phys. Lett. B634, 498 (2006) arXiv:hep-ph/0511328; Z. Nagy, Phys. Rev. D68, 094002 (2003) arXiv:hep-ph/0307268.

[8] G. Corcella et al., JHEP 0101, 010 (2001) arXiv:hep-ph/0011363].

[9] T. Sjostrand, P. Eden, C. Friberg, L. Lonnblad, G. Miu, S. Mrenna and E. Norrbin, Comput. Phys. Commun. 135, 238 (2001) arXiv:hep-ph/0010017.

[10] F. Hautmann and H. Jung, JHEP 0810, 113 (2008) arXiv:0805.1049 [hep-ph]].

[11] M. Ciafaloni, Nucl. Phys. B296, 49 (1988); S. Catani, F. Fiorani and G. Marchesini, Phys. Lett. B234, 339 (1990).

[12] M. Dasgupta and G. P. Salam, Phys. Lett. B512, 323 (2001) arXiv:hep-ph/0104277, JHEP 0203, 017 (2002) arXiv:hep-ph/0203009.

[13] A. Banfi, M. Dasgupta and Y. Delenda, Phys. Lett. B665, 86 (2008) arXiv:0804.3786 [hep-ph]].

[14] G. Bozzi, S. Catani, G. Ferrera, D. de Florian and M. Grazzini, Nucl. Phys. B815, 174 (2009) arXiv:0812.2862 [hep-ph]].

[15] A. Banfi, G. P. Salam and G. Zanderighi, Eur. Phys. J. C47, 113 (2006) arXiv:hep-ph/0601139.

[16] A. Banfi, G. P. Salam and G. Zanderighi, JHEP 0707, 026 (2007) arXiv:0704.2999 [hep-ph]].

[17] T. O. Eynck, E. Laenen and L. Magnea, JHEP 0306, 057 (2003) arXiv:hep-ph/0305179.

[18] A. Banfi, M. Dasgupta and R. M. D. Delgado, arXiv:0906.4277 [hep-ph]. 